\title{
Microphthalmia with brain and digit anomalies
}

INSERM

\section{Source}

INSERM. (1999). Orphanet: an online rare disease and orphan drug data base.

Microphthalmia with brain and digit anomalies. ORPHA:139471

Microphthalmia with brain and digit anomalies is characterised by anophthalmia or microphthalmia, retinal dystrophy, and/or myopia, associated in some cases with cerebral anomalies. It has been described in two families. Polydactyly may also be present. Linkage analysis allowed identification of mutations in the BMP4 gene, which has already been shown to play a role in eye development. 